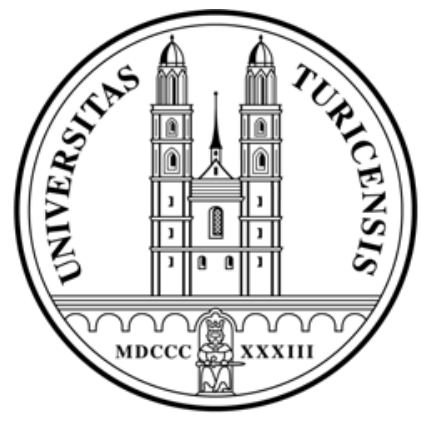

Institute for Empirical Research in Economics

University of Zurich

Working Paper Series

ISSN 1424-0459

Working Paper No. 371

Learning, public good provision, and the information trap

Aleksander Berentsen, Esther Bruegger and Simon Loertscher

May 2008 


\title{
Learning, public good provision, and the information trap*
}

\author{
Aleksander Berentsen† Esther Bruegger ${ }^{\ddagger}$ and Simon Loertscher ${ }^{\S}$ \\ June 22, 2007
}

\begin{abstract}
We consider an an economy where decision maker(s) do not know the true production function for a public good. By using Bayes rule they can learn from experience. We show that the economy may learn the truth, but that it may also converge to an inefficient policy where no further inference is possible so that the economy is stuck in an information trap. We also show that our results are robust with respect to experimentation
\end{abstract}

UNDER CONSTRUCTION

Keywords: Public economics, learning, size of government.

JEL-Classification: D72, H10, D83.

*The paper has benefitted from very insightful comments of two anonymous referees and the editor. We also want to thank Joshua Gans, Roland Hodler, Andy McLennan, Torsten Persson, Fabienne Peter, Ronny Razin, Kurt Schmidheiny, Christian Schultz, Martin Wagner, Volker Wieland and seminar participants at the University of Bern, SSES 2004 in Basel, PCS 2005 in New Orleans, EPCS 2005 in Durham and AEA in Boston 2006 for most valuable discussions and comments. Very special thanks go to Roberto Schonmann. Financial support by the WWZ-Forum Basel is gratefully acknowledged. A previous version of the paper circulated under the title "Learning, voting and the information trap". Any remaining errors are ours.

${ }^{\dagger}$ Economics Department, University of Basel, Petersgraben 51, CH-4003 Basel. eMail: aleksander.berentsen@unibas.ch

${ }^{\ddagger}$ NERA Economic Consulting, 1166 Avenue of the Americas, 34th Floor, New York, NY 10036. eMail: estherbruegger@gmx.net

$\S$ Economics Department, University of Melbourne, Economics \& Commerce Building, Victoria 3010, Australia. eMail: simonl@unimelb.edu.au 
...for after falling a few times they would in the end certainly learn to walk...

Immanuel Kant (1784)

\section{Introduction}

Broadly speaking, there are two theories regarding the effects of government activity on the economy. ${ }^{1}$ Some economists emphasize the crucial role of government in securing property rights, enforcing contracts, providing national security and, perhaps, guaranteeing a moderate minimum income for every one. These proponents do not deny that some government activity is better than none and they would probably argue that at a small scale, public production exhibits very large marginal productivity. These marginal products, however, then decline quickly and eventually become negative. Other economists believe that government is most productive if it operates on a large scale because of increasing returns. According to this view, operating on a small scale, the marginal product of government activity is moderate as it merely serves to appease the poor, yet fails to exhaust their full economic potential. ${ }^{2}$

The question which of these two theories is right cannot be answered by a priory arguments. ${ }^{3}$ However, it is crucial to know whether in the long run efficient policies are

\footnotetext{
${ }^{1}$ See, e.g., Hayek (1944), Hazlitt (1946) and Friedman (1962, 1997) or Rosenstein-Rodan (1943), Myrdal (1975) and Sachs (2005).

${ }^{2}$ This hypothesis is consistent with Acemoglu and Robinson (2000).

${ }^{3}$ Blendon, Benson, Brodie, Morin, Altman, Gitterman, Brossard, and James (1997) conducted an opinion survey showing that there is a substantial gap between economists' and the public's beliefs about how the economy functions. Fuchs, Blinder, and Poterba (1998) report findings from another survey that there are significant differences even among professional economists about policy questions as well as parameter estimates. This can be regarded as evidence of uncertainty about which is the correct model. Bartels (1996) notes that the "[t]he political ignorance of the American voter is one
} 
chosen, i.e., whether experience will eventually lead the economy to learn the truth. This is the question we address in this paper. For this purpose, we construct a model with uncertainty about how the economy functions. The decision maker does not know which of two possible production functions for a public good is the true one. For any given belief, the policy maker maximizes his short-run expected utility and, after observing the tax rate and the level of production of the public good, updates her beliefs using Bayes' rule. We show that in the long run the true production function may be learned, but the economy may also converge to an inefficient policy where no further inference is possible so that the economy is stuck in an information trap. We also show that this result is robust with respect to experimentation.

The paper relates to several strands of literature. First, we investigate the correctness of Kant's optimistic view on the prospects of enlightenment, expressed in the quote above and in the following (Kant, 1784, fourth paragraph): "But that the public should enlighten itself is more likely; indeed, if it is only allowed freedom, enlightenment is almost inevitable." One of the policies adopted in the long-run equilibrium of our model is the Kantian policy. The other policy is non-Kantian in the sense that the economy is stuck in an information trap, where the truth will never be learned. Interestingly, this latter policy can be Pareto inefficient. In this respect, our paper is related to Hess and Orphanides (2001) who investigate the correctness of Kant's perpetual peace hypothesis. ${ }^{5}$ of the best-documented features of contemporary politics, but the political significance of this political ignorance is far from clear."

${ }^{4}$ For a more modern, similarly optimistic view, see Wittman (1989).

${ }^{5}$ They show that Kant's conjecture that a world populated exclusively by democracies generates perpetual peace is correct insofar as perpetual peace is an equilibrium outcome if there are only democratic regimes. However, even such a world does not necessarily imply perpetual peace as there are other equilibria where wars occur with positive probability. 
Second, the paper relates to the political economy literature on heterogenous social beliefs that are consistent with either multiple equilibria or long-run divergence in beliefs, such as Piketty (1995), Spector (2000) and Alesina and Angeletos (2005). ${ }^{6}$ In contrast to Piketty and Spector, in our model all households share the same information and beliefs, but are eventually hindered from learning the truth.An important difference between our model and the one of Alesina and Angeletos is that their equilibria can be ranked unambiguously only from the point of view of the median household. ${ }^{7}$

Third, our paper relates to the literature on learning. Our main finding is related to the well-known result that impatient Bayesian learners can optimally fail to learn the true parameter values (see, e.g., Easley and Kiefer, 1988). ${ }^{8}$ In this strand of literature, the most important predecessor to our paper is McLennan (1984) who studies learning by a monopolistic seller who faces two linear demand functions intersecting at some price and who is uncertain about which of the two is true. ${ }^{9}$ The paper is also related to Laslier, Trannoy, and Van Der Straeten (2003) who study voting over unemployment

\footnotetext{
${ }^{6}$ There is a substantial political economy literature that deals with asymmetric information where one type of player is better informed than another; see, e.g., Crawford and Sobel (1982), Feddersen and Pesendorfer (1996), Schultz (1996) or Heidhues and Lagerlöf (2003). Schultz studies a setting where "voters, but not parties, are uncertain about the functioning of the economy". The effects of extending our model in this direction are discussed in Section 4.2.

${ }^{7}$ Moreover, the sources of multiplicity are quite different. In their model, multiplicity stems from differences in social beliefs about which fraction of income is fair or merited, whereas in ours it arises from incomplete information and incomplete learning.

${ }^{8}$ Insofar as incomplete learning is concerned, a very similar phenomenon obtains in models of herding such as Banerjee (1992) and Bikhchandani, Hirshleifer, and Welch (1992). However, the reasons for incomplete learning are very different in the two types of models.

${ }^{9}$ In every period, the seller in McLennan's model observes whether there is sale or not and updates his beliefs accordingly. Among other things he shows that with positive probability the seller ends up charging the price where the two demand functions intersect, at which point no further learning is possible. A more detailed discussion of the relationship between our model and McLennan's is deferred to the end of section 3 .
} 
benefits when households do not know the (a fortiori unobserved) distribution of skills of the unemployed. They uncover a possibility of inefficiency that is quite similar to our finding. An important contrast is that in our model the dynamics are not monotone. ${ }^{10}$

The remainder of the paper is structured as follows. Section 2 introduces the model. Section 3 analyzes the dynamic learning process. Section 4 extends the model by introducing experimentation by the policy maker. Section 5 concludes. All the proofs are in the Appendix.

\section{The model}

There is a continuum of individuals whose total mass is normalized to one. Individual income $y_{i}$ is distributed according to the density function $f\left(y_{i}\right)$. The mean income is one and the median income is denoted by $y^{m}$. The support of the distribution is $\left[y^{\text {inf }}, y^{\text {sup }}\right]$ with $0 \leq y^{\text {inf }}<y^{\text {sup }}<\infty$. Each individual $i$ derives utility from private consumption $c_{i}$ and from a public good $H(g)$, which is a function of government expenditure $g$. Individual $i$ 's utility is $u_{i}=c_{i}+H(g)$. Note that individuals differ only with respect to their private consumption, but are identical with respect to their valuation of the public good. Since mean income is one, the government's budget constraint is $g=\tau$, where $0 \leq \tau \leq 1$ is a flat tax rate. Accordingly, individual $i$ 's consumption is $c_{i}=(1-\tau) y_{i}$.

We assume that $H(g)$ is twice differentiable, strictly concave and increasing in $g$ for $g$ close to zero. This assumption makes sure that for every household there is a unique bliss

\footnotetext{
${ }^{10}$ The dynamics of our model are more similar to those in Baron (1996), who analyzes voting over public goods programs by a legislature when there is uncertainty about which legislators can make proposals in future periods. As in our model, the economy "hops" towards its absorbing state, which in his model is given by the complete information bliss point of the median voter. In contrast to Baron's model, we have two absorbing states, one of which can be Pareto inefficient.
} 
point tax rate. Using the budget restrictions $g=\tau$ and $c_{i}=(1-\tau) y_{i}$, we can replace $c_{i}$ and $g$ and write $i$ 's utility as a function of the tax rate only, $u_{i}(\tau)=(1-\tau) y_{i}+H(\tau)$. Note that $H(\tau)$ is concave in $\tau$. We denote by $\tau^{i}$ individual $i$ 's optimal tax rate, which is implicitly defined by $\frac{\partial H}{\partial \tau}\left(\tau^{i}\right)=y_{i}$. Since $H(\tau)$ is concave, $\tau^{i}$ is decreasing in $y_{i}$. Thus, the single crossing property is satisfied (see Gans and Smart, 1996; Persson and Tabellini, 2000, ch. 2, condition 2.4). Denote by $\tau^{m}$ the optimal tax rate of the median income household.

We further assume that $H$ and the income distribution satisfy $y^{s u p}<\frac{\partial H}{\partial g}(0)$ and $\frac{\partial H}{\partial g}(y)<y^{i n f}$. Therefore, even the richest individual prefers some government activity to none and even the poorest individual's preferred tax rate is less than one. Put differently, only the tax rates $\tau \in P \equiv\left[\tau^{I}, \tau^{I I}\right]$ with $\tau^{I} \equiv H^{\prime-1}\left(y^{\text {sup }}\right)>0$ and $\tau^{I} \equiv H^{\prime-1}\left(y^{\text {inf }}\right)<1$ will be Pareto efficient.

We consider a decision maker who in each period chooses the tax rate that maximizes the median income household's utility $u^{m}=(1-\tau) y^{m}+H(\tau)$. Two comments are in order here. First, it is easy to construct a model with electoral competition along the lines of Persson and Tabellini (2000, ch. 3) which would yield the median voter equilibrium where both parties choose the tax rate the maximizes the median income household's utility. Second, our learning results also hold if the decision maker chooses a different tax policy. For example, he could choose a tax rate that maximizes the income of the 40th percentile or the 55th percentile. In this sense our approach is more general than a focus on a median voter model. ${ }^{11}$ In particular, in Section 4 we show that our results

\footnotetext{
${ }^{11}$ In a previous version of the paper we also modelled electoral competition. We are thankful to the editor to point out that our stylized Downsian model limited the scope of our results.
} 
hold when the decision maker chooses a random tax policy.

We now introduce two production functions $H_{A}(\tau)$ and $H_{B}(\tau)$ satisfying the assumptions made above. These are supposed to represent the two distinct, commonly held views on the effect of government activity on the economy described above. The decision maker has the initial belief $\alpha_{1}$ that the production function $H_{A}$ is the true one, with $0<\alpha_{1}<1$. In every period $t$, he uses the observed outcomes $h_{t}$ to update his beliefs $\alpha_{t+1}$. Without loss of generality, we assume that $H_{A}(\tau)$ is the true production function.

Let $P_{A} \equiv\left[\tau_{A}^{I}, \tau_{A}^{I I}\right]$ and $P_{B} \equiv\left[\tau_{B}^{I}, \tau_{B}^{I I}\right]$ be the sets of Pareto efficient tax rates associated with the production function $H_{A}$ and $H_{B}$, respectively. Let $\tau_{A}^{m}$ and $\tau_{B}^{m}$ be the optimal tax rates for the median household under $H_{A}$ and $H_{B}$, i.e., $\frac{\partial H_{A}}{\partial \tau}\left(\tau_{A}^{m}\right)=y^{m}$ and $\quad \frac{\partial H_{B}}{\partial \tau}\left(\tau_{B}^{m}\right)=$ $y^{m}$. Note that $\tau_{A}^{m} \in P_{A}$ and $\tau_{B}^{m} \in P_{B}$ and observe that $\tau_{A}^{m}$ can be called the Kantian policy since it is the policy that would be chosen if the true production function was known to the policy maker. Without loss of generality we assume that $\tau_{A}^{m}<\tau_{B}^{m}$.

We assume also that the two functions cross at most once for $\tau>0$. If they do not cross, or cross only at some $\tau \notin\left[\tau_{A}^{m}, \tau_{B}^{m}\right]$, the updating problem is fairly simple, as we shall see in Corollary 2 below. Henceforth, with the exception of this corollary, we focus on the case where the two production functions cross, as depicted in the bottom line of Figure 1.

The production function representing the view that the optimal size of government is small has a shape like $H_{A}$ in the bottom panels in Figure 1. It is very steep when $\tau$ is close to zero, but then flattens quickly and eventually decreases in $\tau$. The production function reflecting the view that government is most efficient if large has a shape similar to $H_{B}$ in the bottom panels in Figure 1, which is not very steep at the origin but flattens 

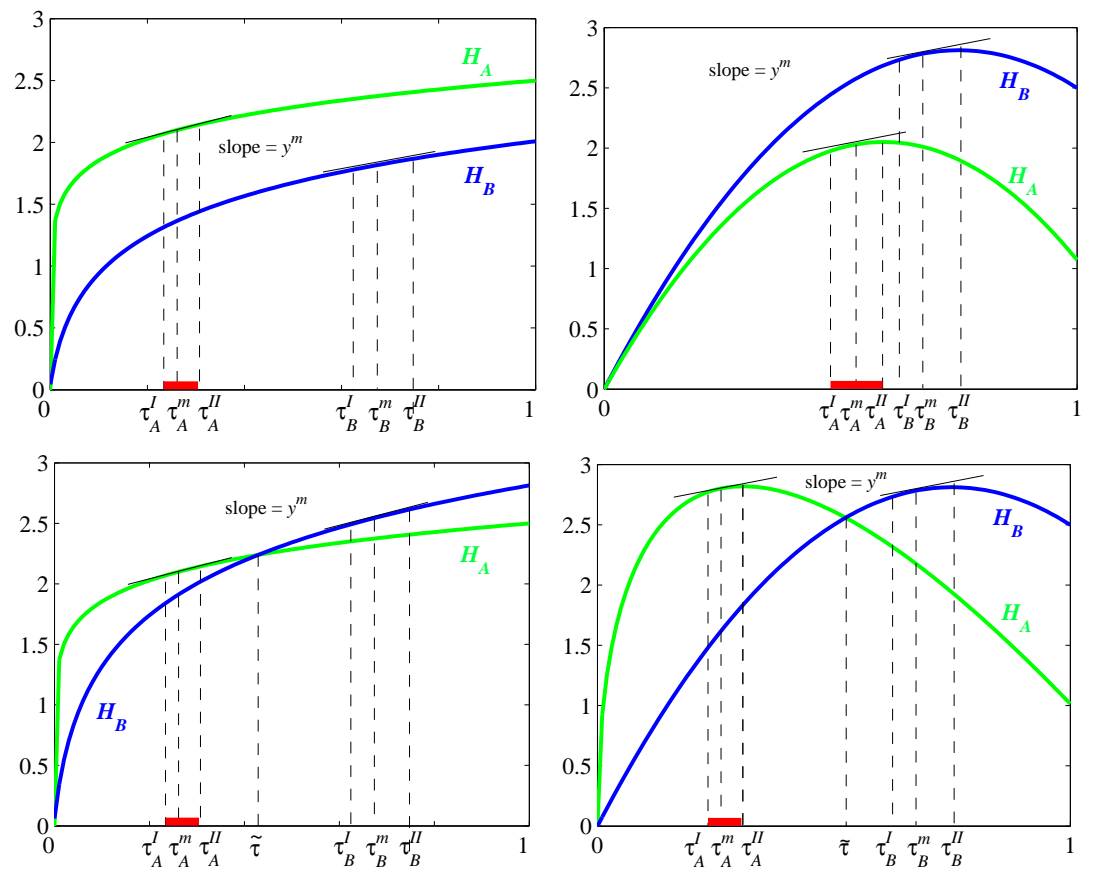

Figure 1: Four variants.

much slower than $H_{A}$. If our sketch of these two opposing views is correct, then the two functions $H_{A}$ and $H_{B}$ will have to intersect at some point, which we denote by $\tilde{\tau}{ }^{12}$

The production of the public good is exposed to uncertainty. If $\tau_{t}$ is the tax rate in period $t$, then the decision maker observes the outcome

$$
h_{t}\left(\tau_{t}, \varepsilon_{t}\right)=H_{A}\left(\tau_{t}\right)+\varepsilon_{t},
$$

where $\varepsilon_{t}$ is an error term drawn randomly in every period. This error term $\varepsilon_{t}$ captures factors influencing the policy outcome except the policy itself. The error terms are normally and independently distributed with mean 0 and variance $\sigma^{2}$; we denote its probability density function by $\phi\left(\varepsilon_{t}\right) .{ }^{13}$ Note that without noise, the learning process,

\footnotetext{
${ }^{12}$ Conceptionally, $\tilde{\tau}$ corresponds to the price in McLennan (1984) where the demand functions intersect.

${ }^{13}$ The normality assumption is only sufficient. As becomes clear from the proof of Proposition 2, all our results will hold for any distribution $f\left(\varepsilon_{t}\right)$ that have full support and whose likelihood ratio $l\left(\varepsilon_{t}\right) \equiv \frac{f\left(H_{B}\left(\tau_{t}\right)+\varepsilon_{t}\right)}{f\left(\varepsilon_{t}\right)}$ is monotone in $\varepsilon_{t}$ and takes on values from zero to infinity.
} 
described below, would be degenerate since one observation would be sufficient to identify the true production function.

In period $t+1$, the entire history $\mathcal{H}_{t} \equiv\left\{\left(h_{j}, \tau_{j}\right)\right\}_{j=1}^{t}$ of previously implemented tax rates and associated policy outcomes is known to the decision maker. Since his belief in period $t$ that $H_{A}$ is the true is $\alpha_{t}$, the expected level of the public good in period $t$ for tax rate $\tau_{t}$ is

$$
H_{t}\left(\tau_{t}\right) \equiv \alpha_{t} H_{A}\left(\tau_{t}\right)+\left(1-\alpha_{t}\right) H_{B}\left(\tau_{t}\right)
$$

\section{Dynamics and long-run equilibria}

We now derive the long-run equilibrium in our model. We assume that in every period $t$ the decision maker maximizes myopically the expected utility of the median households utility $\left(1-\tau_{t}^{m}\right) y^{m}+H_{t}\left(\tau_{t}^{m}\right)$. This assumption is a good approximation if periods are long compared to the patience of households. The learning problem we explore captures the decision problem of a decision maker who faces uncertainty about which of two models of reality is the correct one and whose actions affect both his current period payoff and his future beliefs. The decision maker can be the government of a country, like a (benevolent or malevolent) dictator or a democratically elected president or a monopolistic seller who faces intersecting demand functions. ${ }^{14}$.

Proposition 1 characterizes the decision maker's optimal policy.

Proposition 1 In every period $t$, the decision maker chooses $\tau_{t}^{m} \in\left[\tau_{A}^{m}, \tau_{B}^{m}\right]$, where $\tau_{t}^{m}$ is implicitly defined by $H_{t}^{\prime}\left(\tau_{t}^{m}\right) \equiv y^{m}$.

Figure 2 depicts the policy maker's choice. His initial belief $\alpha_{1}$ is such that the

\footnotetext{
${ }^{14}$ Or an athlete or a student who does not know whether he should practice harder or less hard.
} 
expected production function in period 1 is $H_{1}$. The policy implemented in period 1 is $\tau_{1}^{m}$

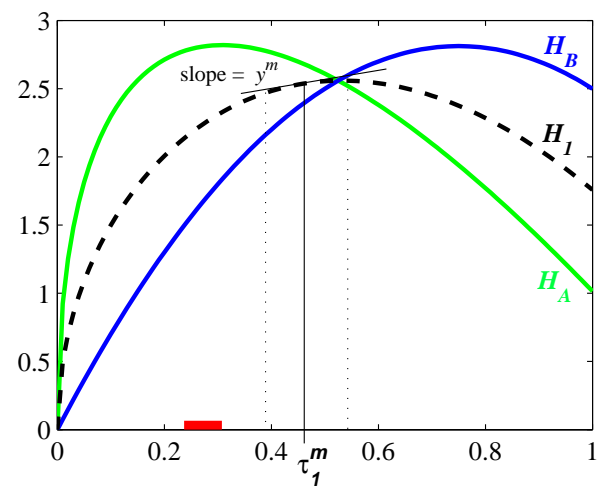

Figure 2: Equilibrium outcome in period 1.
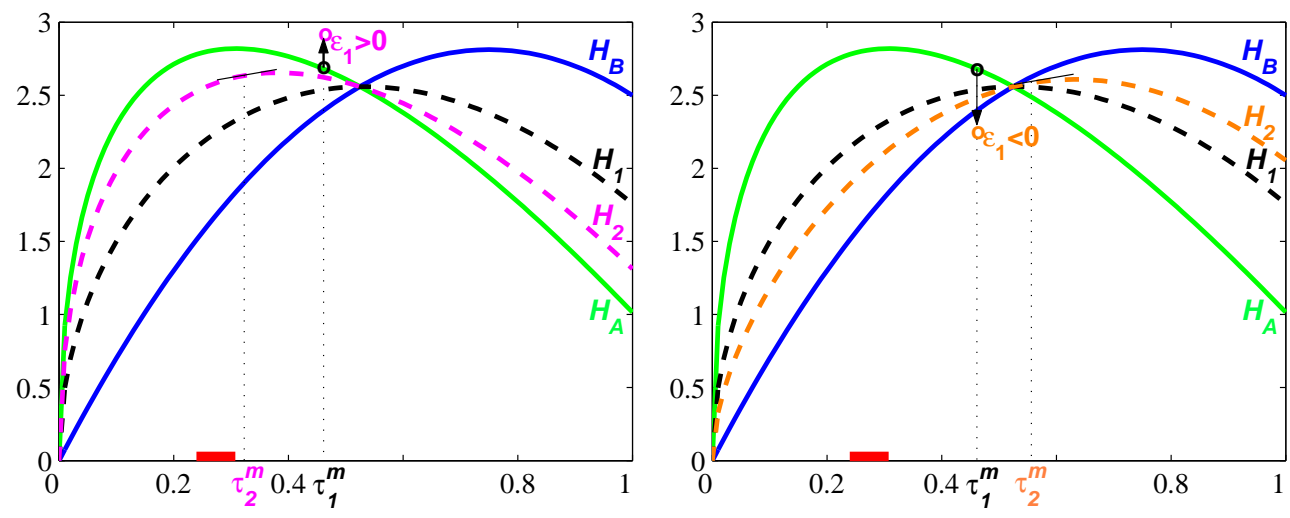

Figure 3: Inferences and outcome in period 2, as a function of $\varepsilon_{1}$.

Figure 3 illustrates the impact of the error term on the decision maker's belief and on the equilibrium tax rate in the next period. After implementing $\tau_{1}^{m}$, the shock $\varepsilon_{1}$ materializes. If $\varepsilon_{1}>0$, the outcome is better than expected under $H_{1}$, and therefore, the updated belief is $\alpha_{2}>\alpha_{1}$ and the new expected production function $H_{2}$ is as shown in the left hand panel. On the other hand, if $\varepsilon_{1}<0$, the outcome is worse than expected under $H_{1}$, and therefore $\alpha_{2}<\alpha_{1}$ yielding $H_{2}$ as shown in the right hand panel. In both cases, the expected production function $H_{2}$ is the basis for equilibrium in period 2 . 
Note that only a strict subset of the feasible tax rates are implemented in equilibrium, i.e., $\tau_{t}^{m} \in\left[\tau_{A}^{m}, \tau_{B}^{m}\right] \subset[0,1]$. This property is illustrated in Figure 4 .

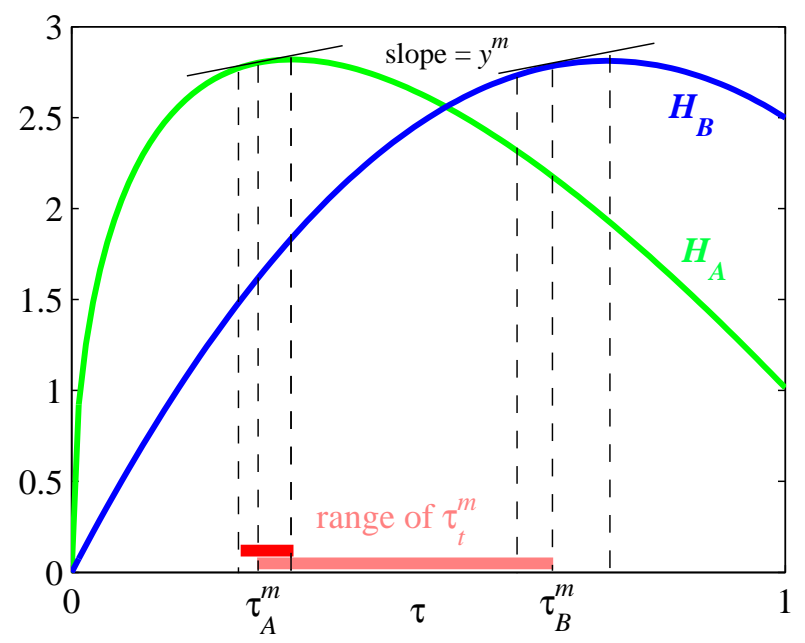

Figure 4: Range of equilibrium tax rates.

\subsection{An informal discussion}

The decision maker's problem is a problem of inference. Recall that $\mathcal{H}_{t} \equiv\left\{\left(h_{i}, \tau_{i}\right)\right\}_{i=1}^{t}$ is the history up to date $t$. Accordingly, let $\operatorname{Pr}\left(H_{A} \mid \mathcal{H}_{t}\right)$ denote the conditional probability that $H_{A}$ is true given history $\mathcal{H}_{t}$. Denote by $\operatorname{Pr}\left(h_{t} \mid H_{A}, \tau_{t}\right)$ the probability of observing $h_{t}$ given that $H_{A}$ is true and given that policy $\tau_{t}$ is implemented. Then, by Bayes rule

$$
\operatorname{Pr}\left(H_{A} \mid \mathcal{H}_{t}\right)=\frac{\operatorname{Pr}\left(H_{A} \mid \mathcal{H}_{t-1}\right) \operatorname{Pr}\left(h_{t} \mid H_{A}, \tau_{t}\right)}{\operatorname{Pr}\left(H_{A} \mid \mathcal{H}_{t-1}\right) \operatorname{Pr}\left(h_{t} \mid H_{A}, \tau_{t}\right)+\left(1-\operatorname{Pr}\left(H_{A} \mid \mathcal{H}_{t-1}\right)\right) \operatorname{Pr}\left(h_{t} \mid H_{B}, \tau_{t}\right)}
$$

Since households are rational, they use Bayes rules (3) to update their beliefs, i.e., $\alpha_{t+1}=$ $\operatorname{Pr}\left(H_{A} \mid \mathcal{H}_{t}\right)$. Since the probability of observing $h_{t}$ is higher under the true production function $H_{A}$ than under $H_{B}, \alpha_{t+1}$ should be expected to converge to one as the number of observations gets large. However, recall that the two production functions intersect at $\tilde{\tau}$ which implies that $\operatorname{Pr}\left(h_{t} \mid H_{A}, \tilde{\tau}\right)=\operatorname{Pr}\left(h_{t} \mid H_{B}, \tilde{\tau}\right)$. Inspection of (3) reveals that in this case, $\alpha_{t+1}=\alpha_{t}$. The observation $h_{t}$ is equally likely under production function $H_{A}$ 
as under $H_{B}$. In this case, the learning process comes to a halt. Let $\tilde{\alpha}$ be the belief such that in equilibrium $\tilde{\tau}$ is implemented. That is, $\tilde{\alpha}$ solves $\tilde{\alpha} H_{A}^{\prime}(\tilde{\tau})+(1-\tilde{\alpha}) H_{B}^{\prime}(\tilde{\tau})=y^{m}$, where $\tilde{\tau}$ is such that $H_{A}(\tilde{\tau})=H_{B}(\tilde{\tau})$. Clearly, $\tilde{\alpha} \in(0,1)$ exists and is unique.

\subsection{The information trap}

We now state our main result:

Proposition 2 Let the two production functions cross at some $\tilde{\tau} \in\left(\tau_{A}^{m}, \tau_{B}^{m}\right)$. Then, the only policies that can be implemented in a long-run equilibrium are $\tilde{\tau}$ and $\tau_{A}^{m}$. Formally, a random variable $\tau_{\infty} \in[0,1]$ exists such that $(i) \tau_{t}^{m}$ converges to $\tau_{\infty}$ almost surely as $t$ becomes arbitrarily large, and (ii) the support of $\tau_{\infty}$ is $\left\{\tilde{\tau}, \tau_{A}^{m}\right\}$.

The content of Proposition 2 is that the policy converges to a random variable whose support is $\tilde{\tau}$ and $\tau_{A}^{m}$. This is equivalent to saying that the process of beliefs converges to a random variable whose support consists solely of $\tilde{\alpha}$ and 1 . The result in Proposition 2 is similar to the finding of Hess and Orphanides (2001): What Kant conjectured enlightenment being inevitable in our case, perpetual peace in their case - is indeed an equilibrium outcome, but it is not the only equilibrium outcome.

The reason why there is a range around $\tilde{\tau}$ from which the policy can eventually not escape is that the two production functions have very similar values in the neighborhood of $\tilde{\tau}$. The closer one gets to $\tilde{\tau}$, the less distinguishable the true and the false production function become. Once one is close enough to $\tilde{\tau}$, it thus becomes very difficult to learn anything. Hence, the economy becomes stuck with its current beliefs once these are sufficiently close to $\tilde{\alpha}$, as a consequence of which policy will not change anymore. Hence, one can speak of an information trap around $\tilde{\tau}$. 
Proposition 2 states that the economy converges to either $\tau_{A}^{m}$ or $\tilde{\tau}$. If $\tau_{A}^{I I}<\tilde{\tau}<\tau_{B}^{I}$ the Pareto sets of $H_{A}$ and $H_{B}$ are disjoint and $\tilde{\tau}$ lies in between them, i.e. is Pareto inefficient. The conditions for this require that $H_{A}$ and $H_{B}$ are sufficiently different. From now on we assume that $\tilde{\tau}$ is Pareto inefficient.

Corollary 1 If $\tau_{A}^{I I}<\tilde{\tau}<\tau_{B}^{I}$, then the economy can converge to a Pareto inefficient policy.

That is, if $\tau_{A}^{I I}<\tilde{\tau}<\tau_{B}^{I}$ holds, then learning the truth is particularly relevant as failure to do so implies that policy implemented in the long-run equilibrium is Pareto inefficient.

Proposition 2 has a corollary that follows almost immediately.

Corollary 2 If the two production functions do not cross on $\left[\tau_{A}^{m}, \tau_{B}^{m}\right]$, then $\tau_{t}^{m}$ converges almost surely to $\tau_{A}^{m}$ as $t$ goes to infinity.

Two comments are in order. First, from the proof of Proposition 2 it is clear that all our results go through if $H_{B}$ for $\tau$ close to zero as long $H_{B}$ is concave for all $\tau \geq \tau_{A}^{m}{ }^{15}$ Second, Corollary 2 is probably not surprising. If the two models never make the same predictions over the relevant interval $\left[\tau_{A}^{m}, \tau_{B}^{m}\right]$, households will ultimately learn the truth.

\subsection{The efficiency potential}

Next we present an analytical result for the lower bound of the probability that the policy converges to $\tau_{A}^{m}$. For that purpose, we define the efficiency potential as this minimal probability, which we denote as $\xi$. That is, $\xi \equiv \inf \operatorname{Pr}\left(\lim _{t \rightarrow \infty} \tau_{t} \rightarrow \tau_{A}^{m} \mid \alpha_{1}, \tilde{\tau}\right)$.

\footnotetext{
${ }^{15}$ This guarantees in particular that the function $s \equiv H_{A}-H_{B}$ satisfies $s^{\prime}(\tau)<0$ for all $\tau \in\left[\tau_{A}^{m}, \tau_{B}^{m}\right]$.
} 
Proposition 3 The efficiency potential is strictly smaller than one and, if positive, increases in the quality of the initial belief $\alpha_{1}$ and decrease in $\tilde{\alpha}$. Formally,

$$
\xi=\max \left\{0, \frac{\alpha_{1}-\tilde{\alpha}}{\alpha_{1}(1-\tilde{\alpha})}\right\} \quad \text { and } \quad \frac{\partial \xi}{\partial \alpha_{1}}>0, \frac{\partial \xi}{\partial \tilde{\alpha}}<0 \quad \text { for } \quad \xi>0 .
$$

The fact that $\frac{\partial \xi}{\partial \alpha_{1}}>0$ is very intuitive since one naturally expects a decision maker who is initially better informed to be more likely to adopt the correct belief in the longrun. The sign of the derivative $\frac{\partial \xi}{\partial \tilde{\alpha}}<0$ is also intuitive, but understanding it requires a moment's reflection. For a given $\alpha_{1}>\tilde{\alpha}$, a series of bad shocks is required for the beliefs to be downgraded to $\tilde{\alpha}$. Obviously, as $\tilde{\alpha}$ decreases, a longer series of bad shocks is required for beliefs to be downgraded to $\tilde{\alpha}$. Since a longer series of bad shocks is less likely, the efficiency potential increases as $\tilde{\alpha}$ decreases.

\subsection{McLennan's model}

As metioned in the introduction our model of learning is closely related to McLennan (1984). Let us therefore discuss the similarities and differences. The two models are very similar in that both assume that there is a policy ( $\tilde{\tau}$ in our model, $a$ in McLennan's; for simplicity, we discuss both models using our notation) that, once taken, will inhibit any further inference. The main difference between the two resides in the nature of the random variable, which is binary (sale, no sale) in McLennan's and continuous in our model. The simpler structure allows McLennan to derive the result that under certain restrictions the seller's belief $\alpha$ never jumps over $\tilde{\alpha}$. That is, if he starts with $\alpha_{1}<\tilde{\alpha}$, his long run belief $\alpha_{\infty}$ will be either 0 or $\tilde{\alpha}$ and if he starts with $\alpha_{1}>\tilde{\alpha}$ it will be either $\tilde{\alpha}$ or 1 . No such result obtains in our model because for any given $\alpha_{t} \in(0, \tilde{\alpha})$ there is 
always a positive probability that a shock occurs such that $\alpha_{t+1}>\tilde{\alpha}{ }^{16}$

One important consequence of this is that we cannot use McLennan's (1984, p. 3434) arguments to establish analytically that $\tilde{\alpha}$ is reached with positive probability. To see this, observe first that our Proposition 3 is actually a statement conditional on $H_{A}$ being true. Without this condition, it would read: The support of $\tau_{\infty}$ is $\left\{\tau_{B}^{m}, \tilde{\tau}, \tau_{A}^{m}\right\}$, or in terms of beliefs, the support of $\alpha_{\infty}$ is $\{0, \tilde{\alpha}, 1\}$. Second, denote by $p_{0}(\alpha), \tilde{p}(\alpha)$ and $p_{1}(\alpha)$ the unconditional probability of converging to the absorbing state $0, \tilde{\alpha}$ and 1 , respectively. Since $p_{0}(\alpha), \tilde{p}(\alpha)$ and $p_{1}(\alpha)$ are probabilities and because all paths converge,

$$
p_{0}(\alpha)=1-\tilde{p}(\alpha)-p_{1}(\alpha)
$$

Then because of the elementary property of Bayesian updating that the expected posterior is equal to the prior,

$$
p_{0}(\alpha) 0+\tilde{p}(\alpha) \tilde{\alpha}+p_{1}(\alpha)=\alpha
$$

In contrast to McLennan, who has the additional restriction that for, say, $\alpha<\tilde{\alpha}$ the only absorbing states are $\{0, \tilde{\alpha}\}$, the system of the two equations (4) and (5) with three unknowns is indeterminate. Without additional restrictions, neither $\tilde{p}=0$ nor $\tilde{p}>0$ can be ruled out. Therefore, it is not possible to prove that $\tilde{p}(\alpha)>0$ along the lines in McLennan (p. 343-4), as suggested by one careful reader.

\subsection{Numerical results}

The distribution of the long-run beliefs $\alpha_{\infty}$ cannot be calculated explicitly. We therefore have to rely on simulations in order to approximate the probability that beliefs converge

\footnotetext{
${ }^{16}$ To see this, observe that $\alpha_{t+1}\left(\varepsilon_{t}\right)=\frac{\alpha_{t}}{\alpha_{t}+\left(1-\alpha_{t}\right) l\left(\varepsilon_{t}\right)}$, where $l\left(\varepsilon_{t}\right)$ is the likelihood ratio that as a function of the shock $\varepsilon_{t}$ can take any value between zero and infinity. Consequently, $\alpha_{t+1}$ is a random variable with support $(0,1)$.
} 
to $\alpha_{\infty}=\tilde{\alpha}$ and $\alpha_{\infty}=1$, respectively. Our simulations suggest that convergence to $\tilde{\tau}$ occurs for a wide range of initial conditions. This is of particular interest because $\tilde{\tau}$ can be Pareto inefficient (Corollary 1).

The simulation results are collected in the two tables below for two different constellations of production functions. ${ }^{17}$ Figure 5 shows three functions which are taken as the production function of the public good. For Table 1, we use the blue function $\left(H_{A}\right)$ as the true production function, and the green function $\left(H_{G}\right)$ as the alternative production function. For Table 2, again the blue function $\left(H_{A}\right)$ is the true production function and the red one $\left(H_{R}\right)$ is the alternative. An entry in the table is the share of draws for which

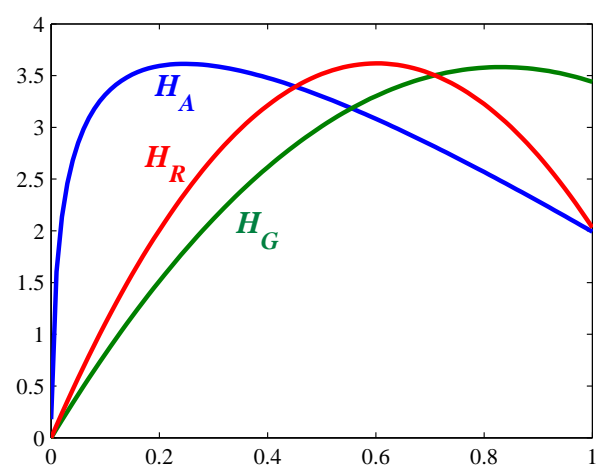

Figure 5: The functions used for the simulations reported in Tables 1 and 2.

the belief converged to 1 for a given combination of initial belief $\alpha_{1}$ and noise $\sigma$. For every entry we did a hundred draws. One minus the table entry gives the share of draws that converged to the inefficient tax rate. ${ }^{18}$ For example, the 1 in the top left entry of Table 1 means that for $\alpha_{1}=0.1$ and $\sigma=0.2$ every draw converged to 1 , for the blue (true) and green (untrue) production function. Note that the smaller $\sigma$, the higher the probability of reaching $\tau_{A}^{m}$. This is intuitive because a smaller variance of the shocks

\footnotetext{
${ }^{17}$ All Matlab-files are available at http://www.wwz.unibas.ch/witheo/aleks/.

${ }^{18}$ Note that, as claimed in Proposition 2, all draws either converge to $\tau_{A}^{m}$ or to $\tilde{\tau}$.
} 


\begin{tabular}{|c|c|c|c|c|c|}
\hline \hline $\begin{array}{c}H_{A} \text { and } H_{G} \\
\tilde{\alpha}=0.47\end{array}$ & $\sigma=0.2$ & $\sigma=0.5$ & $\sigma=1$ & $\sigma=2$ & $\xi$ \\
\hline \hline$\alpha_{1}=0.1$ & 1 & 0.99 & 0.21 & 0.01 & 0 \\
\hline$\alpha_{1}=0.2$ & 1 & 0.98 & 0.26 & 0.02 & 0 \\
\hline$\alpha_{1}=0.3$ & 1 & 0.98 & 0.24 & 0 & 0 \\
\hline$\alpha_{1}=0.4$ & 1 & 0.99 & 0.21 & 0 & 0 \\
\hline$\alpha_{1}=0.5$ & 1 & 0.97 & 0.25 & 0.10 & 0.12 \\
\hline$\alpha_{1}=0.6$ & 1 & 1 & 0.57 & 0.48 & 0.42 \\
\hline$\alpha_{1}=0.7$ & 1 & 0.99 & 0.76 & 0.67 & 0.62 \\
\hline$\alpha_{1}=0.8$ & 1 & 1 & 0.94 & 0.76 & 0.78 \\
\hline$\alpha_{1}=0.9$ & 1 & 1 & 0.91 & 0.92 & 0.90 \\
\hline
\end{tabular}

Table 1: Results when $H_{A}$ is true and $H_{G}$ is the alternative.

\begin{tabular}{|c|c|c|c|c|c|}
\hline \hline $\begin{array}{c}H_{A} \text { and } H_{R} \\
\tilde{\alpha}=0.52\end{array}$ & $\sigma=0.2$ & $\sigma=0.5$ & $\sigma=1$ & $\sigma=2$ & $\xi$ \\
\hline \hline$\alpha_{1}=0.1$ & 0.99 & 0.36 & 0.01 & 0 & 0 \\
\hline$\alpha_{1}=0.2$ & 1 & 0.29 & 0 & 0 & 0 \\
\hline$\alpha_{1}=0.3$ & 1 & 0.28 & 0 & 0 & 0 \\
\hline$\alpha_{1}=0.4$ & 1 & 0.29 & 0 & 0 & 0 \\
\hline$\alpha_{1}=0.5$ & 1 & 0.21 & 0 & 0 & 0 \\
\hline$\alpha_{1}=0.6$ & 1 & 0.58 & 0.31 & 0.29 & 0.27 \\
\hline$\alpha_{1}=0.7$ & 1 & 0.75 & 0.59 & 0.58 & 0.53 \\
\hline$\alpha_{1}=0.8$ & 1 & 0.92 & 0.76 & 0.71 & 0.73 \\
\hline$\alpha_{1}=0.9$ & 1 & 0.96 & 0.91 & 0.90 & 0.88 \\
\hline
\end{tabular}

Table 2: Results when $H_{A}$ is true and $H_{R}$ is the alternative. 
increases the informativeness of the policy outcome.

Three further remarks are in order. First, the efficiency potential $\xi$ has some bite indeed. For $\sigma=2, \xi$ is quite close to the numerical results both in Table 1 and 2. Thus, $\xi$ is not a merely theoretical lower bound. Second, the difference between the numerical results and the efficiency potential for $\sigma=2$ and $\alpha_{1}=0.5$ in Table 1 and for $\alpha_{1}=0.8$ in Table 2 is not statistically significant. ${ }^{19}$ Third, consider the columns for $\sigma=1$ in Table 1 and $\sigma=0.5$ in Table 2 to see that the probability of convergence to the good policy does not increase monotonically in the initial belief $\alpha_{1} \cdot{ }^{20}$ The intuition for this behavior seems to be that starting from very bad initial beliefs, i.e. $\alpha_{1}$ close to zero, increases in $\alpha_{1}$ may well increase the likelihood of adopting a good policy in the long-run. However, as $\alpha_{1}$ becomes larger it gets closer to $\tilde{\alpha}$ and thereby increases the probability of adopting a bad policy in the long-run. Witness in particular that the minima in these columns are reached for the $\alpha_{1}$ closest to, and to the left of, $\tilde{\alpha}$, which are, respectively, $\alpha_{1}=0.4$ and $\alpha_{1}=0.5$.

\section{Experimentation}

There are many directions in which our model can be extended. Here, we consider experimentation which is particularly relevant. There are two reasons for doing so. First, it demonstrates by example that our results also hold in a model where the decision

\footnotetext{
${ }^{19}$ The standard errors are 0.03 and 0.05 . The complete simulation data and the tables augmented with standard errors are available at http://www.wwz.unibas.ch/witheo/aleks/.

${ }^{20}$ In Table 1, the probability decreases from $0.26(0.04)$ to $0.21(0.04)$, while in Table 2 it decreases from $0.36(0.05)$ to $0.21(0.04)$, where standard errors are in parentheses. Thus, the difference between 0.36 and 0.21 in Table 2 is statistically significant whereas the difference between 0.26 and 0.21 in Table 1 is not.
} 
maker is not choosing the tax rate which is optimal for the median household. Second, so far we have assumed that the decision maker behaves myopically. This is in particular questionable if the economy is stuck in the information trap since the decision maker knows that reaching $\tilde{\tau}$ is bad: it prevents learning with probability one. It is thus of particular relevance to check whether the result that the economy may end up in an information trap breaks down when we endow the decision maker with some forward looking ability. We do this by allowing him do experiments.

A plausible, and feasible, way of modelling foresighted and experimenting behavior is the following. In any period $t$, let the decision maker choose $\tau_{t}^{m}-\Delta$ and $\tau_{t}^{m}+\Delta$, where $\Delta>0$ measures the degree of foresightedness and $\tau_{t}^{m}$ is the still myopic bliss point tax rate of the median household. The implemented policy is determined by flipping a fair coin. ${ }^{21}$ The larger $\Delta$, the greater the degree of foresightedness and/or the larger the willingness to experiment.

The question that interests us is whether in the long-run beliefs converge towards $\alpha_{\infty}=\tilde{\alpha}$ with positive probability. To answer this question, we use again simulations. The results are collected in Table 3 . The true production function $H_{A}$ and the alternative $H_{G}$ are the same as in Table 1 above. We set $\sigma=1$ and let $\alpha_{1}$ increase from 0.1 to 0.9 , while $\Delta$ increases from 0.00001 to 0.1 . (The first column with $\Delta=0$ is a reprint from Table 1.) For each pair $\left(\alpha_{1}, \Delta\right)$, we ran 100 draws. Table entries give the number of draws for which the process ended with beliefs $\alpha_{\infty}=1$. In this case, the good policy $\tau_{A}^{m}$

\footnotetext{
${ }^{21}$ Alternatively, one could have a percentage experimentation, according to which positions would be $(1-\Delta) \tau_{t}^{m}$ and $(1+\Delta) \tau_{t}^{m}$. The assumption that randomization is fifty-fifty is made for convenience. We expect the results to be robust to other distributions as long as these are not too skewed towards the true production function.
} 
is implemented in the long run. ${ }^{22}$

\begin{tabular}{|c|c|c|c|c|c|c|}
\hline \hline $\begin{array}{c}H_{A} \text { and } H_{G} \\
\tilde{\alpha}=0.47 \\
\sigma=1\end{array}$ & $\Delta=$ & $\Delta=$ & $\Delta=$ & $\Delta=$ & $\Delta=$ & $\Delta=$ \\
\hline$\alpha_{1}=0.1$ & 0.21 & 0.43 & 0.68 & 0.95 & 1 & 1 \\
\hline$\alpha_{1}=0.2$ & 0.26 & 0.43 & 0.66 & 0.95 & 0.99 & 1 \\
\hline$\alpha_{1}=0.3$ & 0.24 & 0.43 & 0.68 & 0.94 & 0.99 & 1 \\
\hline$\alpha_{1}=0.4$ & 0.21 & 0.36 & 0.67 & 0.94 & 0.99 & 1 \\
\hline$\alpha_{1}=0.5$ & 0.25 & 0.48 & 0.74 & 0.94 & 0.99 & 1 \\
\hline$\alpha_{1}=0.6$ & 0.57 & 0.69 & 0.85 & 0.97 & 1 & 1 \\
\hline$\alpha_{1}=0.7$ & 0.76 & 0.79 & 0.90 & 0.99 & 1 & 1 \\
\hline$\alpha_{1}=0.8$ & 0.94 & 0.93 & 0.97 & 1 & 1 & 1 \\
\hline$\alpha_{1}=0.9$ & 0.91 & 0.97 & 0.99 & 1 & 1 & 1 \\
\hline
\end{tabular}

Table 3: Simulation results for the model with experimentation.

We observe the following. First, if $\Delta$ is sufficiently large, in particular larger than 0.1, then the truth is always learnt in the long-run. Note that $\Delta=0.1$ implies a difference between the policy platforms of 10 percentage points. Even with $\Delta=0.01$, convergence to the Pareto efficient policy is still almost universal. However, as $\Delta$ becomes smaller, the probability of convergence to $\alpha_{\infty}=1$ decreases, too. For example, for $\Delta=0.0001$ and $\alpha_{1} \leq 0.4$, less than 70 out of the 100 draws converged to $\alpha_{\infty}=1$. Of course, $\Delta=0.0001$ corresponds to one percent of a percent and is thus admittedly a very small policy difference. Nonetheless, it represents a positive amount of experimentation and reflects at least some degree of forward looking behavior. Hence, we conclude that our information trap result does not break down 'as soon as even the slightest degree of nonmyopic behavior or experimentation is allowed for', as one reader conjectured. Second,

\footnotetext{
${ }^{22}$ Obviously, this is true only in an approximate sense because given our modelling assumptions experimentation continues even with $\alpha_{\infty}=1$.
} 
consider the column with $\Delta=0.0001$ to see that the non-monotonicity in $\alpha_{1}$ observed above carries over to the model with experimentation. ${ }^{23}$

\section{Conclusions}

We consider an economy where there is uncertainty about how the economy functions. In every period, the decision maker implements a policy. Observations of policies and economic outcomes are used to update the decision makers beliefs, which then serve as the basis for decision making in the following period. We show that the economy can end up in an information trap where no further learning is possible. This result is robust with respect to the introduction of experimentation.

Putnam (1993) has raised the question why some governments fail and others succeed. He explains the failure and success of democracies by referring to differences in political institutions and attitudes. We have provided an alternative explanation why initially identical societies may differ in the long run and more specifically, why some countries may adopt Pareto inferior policies even in the long run. Our explanation is that decision makers face uncertainty and that uncertainty can only be unravelled by experience. Initially identical countries may end up with different outcomes because in combination with bad luck the equilibrium may impede further inferences, so that the uncertainty is never abolished. ${ }^{24}$ Since in our model economies may fail to converge to

\footnotetext{
${ }^{23}$ Though the $\Delta$ 's for which non-convergence to $\tau_{A}^{m}$ is obtained appear rather small, it should be noted that the size of $\Delta$ is only meaningful in relation to the slopes of $H_{A}$ and $H_{B}$ in the neighborhood of $\tilde{\tau}$ : The smaller the difference in these slopes, the larger $\Delta$ can be for non-convergence to occur with positive probability. An example is available upon request/in the Webappendix.

${ }^{24}$ Among other things, we have shown that initial beliefs may be crucial for the long run political outcome. This may help better understand the economic and political difficulties former colonies face who may have been endowed with bad initial beliefs at the time of independence, as emphasized, e.g.,
} 
Pareto efficient policies as a consequence of bad shocks, its predictions are consistent with the observations of Easterly (2001), who notes that some countries' meager growth performance may be caused by bad luck.

\section{Appendix}

Proof of Proposition 1 Since $H_{A}$ and $H_{B}$ are concave, $H_{t}\left(\tau_{t}\right)$ is concave. For any concave function and beliefs $\alpha_{t}$, the distribution function for $\tau_{t}^{i}$ can be derived using standard techniques for the transformation of random variables. ${ }^{25}$ Let $\tau_{t}^{i}=\kappa\left(y_{i}\right)$ denote the inverse of the function $y_{i}=H_{t}^{\prime}\left(\tau_{t}^{i}\right)$ derived from the optimality condition $\frac{\partial H\left(\tau^{i}\right)}{\partial \tau}=y_{i}$ of the model without uncertainty. Since $H_{t}^{\prime \prime}\left(\tau_{t}^{i}\right)$ exists, $\frac{d y_{i}}{d \tau_{t}^{i}}=H_{t}^{\prime \prime}\left(\tau_{t}^{i}\right)$. If we denote by $\Omega\left(\tau_{t}^{i}\right)$ the distribution of $\tau_{t}^{i}$, then the density $\omega\left(\tau_{t}^{i}\right)$ of $\Omega\left(\tau_{t}^{i}\right)$ is given by $\omega\left(\tau_{t}^{i}\right)=$ $f\left(\kappa\left(\tau_{t}^{i}\right)\right)\left|\frac{d y_{i}}{d \tau_{t}^{i}}\right|$, where $\left|\frac{d y_{i}}{d \tau^{i}}\right|$ denotes the absolute value of the derivative $\frac{d y_{i}}{d \tau_{t}^{i}}=H_{t}^{\prime \prime}\left(\tau_{t}^{i}\right)$. Consequently, the optimal tax rate of the voter with the median income is the median optimal tax rate. In any period $t$ the median household's optimal tax rate under the expected production function $H_{t}\left(\tau_{t}\right)$ defined in $(2)$ is implemented in equilibrium. Since by definition $\frac{\partial H_{A}}{\partial \tau}\left(\tau_{A}^{m}\right)=\frac{\partial H_{B}}{\partial \tau}\left(\tau_{B}^{m}\right)$ and since $H_{A}(\tau)$ and $H_{B}(\tau)$ are both concave, we know that $\frac{\partial H_{A}}{\partial \tau}>y^{m}$ and $\frac{\partial H_{B}}{\partial \tau}>y^{m}$ for all $\tau<\tau_{A}^{m}$. Hence, since $\alpha_{t} \leq 1$ for all $t, \tau_{t}^{m} \geq \tau_{A}^{m}$ for all $t$ follows. Symmetric arguments can be applied to rule out $\tau_{t}^{m}>\tau_{B}^{m}$.

Proof of Proposition 2 We prove Proposition 2 by showing that the decision maker's belief $\alpha_{t}$ converges to a random variable $\alpha_{\infty}$ almost surely. From Proposition 1 we then get the convergence result for $\tau_{t}^{m}$.

by Bauer (1981).

${ }^{25}$ See, e.g., Hogg and Craig (1995). 
We first define the function $s(\tau) \equiv H_{A}(\tau)-H_{B}(\tau) \quad$ for $\quad \tau \in\left[\tau_{A}^{m}, \tau_{B}^{m}\right]$. The fact that $s^{\prime}(\tau)<0$ for $\tau \in\left[\tau_{A}^{m}, \tau_{B}^{m}\right]$ is readily established, using $H_{A}^{\prime}(\tau)<H_{B}^{\prime}(\tau)$ for $\tau \in\left[\tau_{A}^{m}, \tau_{B}^{m}\right]$, which follows from concavity of both $H_{A}$ and $H_{B}$ and the fact that $H_{A}^{\prime}\left(\tau_{A}^{m}\right)=H_{B}^{\prime}\left(\tau_{B}^{m}\right)$. Note that for $\tilde{\tau} \in\left[\tau_{A}^{m}, \tau_{B}^{m}\right], s(\tilde{\tau})=0$. Therefore, $s\left(\tau_{A}^{m}\right)>0$ and $s\left(\tau_{B}^{m}\right)<0$.

Let us also define the function $\tau^{m}\left(\alpha_{t}\right)$, which is the tax rate solving the equation in Proposition 1 as a function of the beliefs $\alpha_{t}$. So for a given belief $\alpha_{t}$ we have $\tau_{t}^{m}=$ $\tau^{m}\left(\alpha_{t}\right)$, the unique optimal tax rate of the median voter. Using the implicit function theorem, we have $\frac{\partial \tau_{t}^{m}}{\partial \alpha_{t}}=\frac{-s^{\prime}\left(\tau_{t}^{m}\right)}{\alpha_{t} H_{A}^{\prime \prime}\left(\tau_{t}^{m}\right)+\left(1-\alpha_{t}\right) H_{B}^{\prime \prime}\left(\tau_{t}^{m}\right)}<0$, since $-s^{\prime}>0$ and $\alpha_{t} H_{A}^{\prime \prime}+(1-$ $\left.\alpha_{t}\right) H_{B}^{\prime \prime}<0$ by concavity. This is also quite intuitive. As the beliefs that $H_{A}$ is true increase, the equilibrium tax rate decreases, i.e., is closer to $\tau_{A}^{m}$. Finally, let us define $w\left(\alpha_{t}\right) \equiv s\left(\tau^{m}\left(\alpha_{t}\right)\right)$, which gives us the difference between the two production function in equilibrium as a function of the beliefs in period $t$. The function $w$ is defined on the interval $[0,1]$. The fact that $\frac{\partial w}{\partial \alpha_{t}}=s^{\prime} \tau^{m \prime}>0$ follows immediately from the above observations. Moreover, because with $\tilde{\tau} \in\left[\tau_{A}^{m}, \tau_{B}^{m}\right], s(\tilde{\tau})=0$, we have $w(\alpha(\tilde{\tau}))=0$ for a unique $\tilde{\alpha} \in(0,1)$ and $-\infty<w(0)<0<w(1)<\infty$.

Let $\alpha_{1}=\operatorname{Pr}\left(H_{A}\right)$ and $1-\alpha_{1}=\operatorname{Pr}\left(H_{B}\right)$ be the exogenously given prior beliefs that $H_{A}$ and $H_{B}$ are true, respectively, and let

$\operatorname{Pr}\left(h_{t} \mid H_{A}\right)=\phi\left(h_{t}-H_{A}\left(\tau_{t}\right)\right)=\phi\left(\varepsilon_{t}\right) \quad$ and $\operatorname{Pr}\left(h_{t} \mid H_{B}\right)=\phi\left(h_{t}-H_{B}\left(\tau_{t}\right)\right)=\phi\left(w\left(\alpha_{t}\right)+\varepsilon_{t}\right)$,

be the respective probabilities of observing $h_{t}$ when $H_{A}$ and when $H_{B}$ is true, where $\phi($.$) is the density of the normal with mean zero and variance \sigma^{2}{ }^{26}$ After history $\mathcal{H}_{t}=$

\footnotetext{
${ }^{26}$ Note that for a continuous random variable any single observation has probability zero. Nonetheless, L'Hôpital's rule can be used to determine to posterior probability, so that the density rather than the cdf is appropriate.
} 
$\left\{\left(h_{i}, \tau_{i}\right)\right\}_{i=1}^{t}$, the period $t+1$ belief can be written as

$\alpha_{t+1}=\frac{1}{1+\frac{\operatorname{Pr}\left(H_{B}\right) \operatorname{Pr}\left(h_{1} \mid H_{B}\right) \operatorname{Pr}\left(h_{2} \mid H_{B}\right) \ldots \operatorname{Pr}\left(h_{t} \mid H_{B}\right)}{\operatorname{Pr}\left(H_{A}\right) \operatorname{Pr}\left(h_{1} \mid H_{A}\right) \operatorname{Pr}\left(h_{2} \mid H_{A}\right) \ldots \operatorname{Pr}\left(h_{t} \mid H_{A}\right)}}=\frac{1}{1+\frac{\left(1-\alpha_{1}\right) \phi\left(w\left(\alpha_{1}\right)+\varepsilon_{1}\right) \phi\left(w\left(\alpha_{2}\right)+\varepsilon_{2}\right) \ldots \phi\left(w\left(\alpha_{t}\right)+\varepsilon_{t}\right)}{\alpha_{1} \phi\left(\varepsilon_{1}\right) \phi\left(\varepsilon_{2}\right) \ldots \phi\left(\varepsilon_{t}\right)}}$.

Define

$$
N_{t+1} \equiv \frac{\left(1-\alpha_{1}\right) \phi\left(w\left(\alpha_{1}\right)+\varepsilon_{1}\right) \phi\left(w\left(\alpha_{2}\right)+\varepsilon_{2}\right) \cdot \ldots \cdot \phi\left(w\left(\alpha_{t}\right)+\varepsilon_{t}\right)}{\alpha_{1} \phi\left(\varepsilon_{1}\right) \phi\left(\varepsilon_{2}\right) \cdot \ldots \cdot \phi\left(\varepsilon_{t}\right)},
$$

such that (6) becomes $\alpha_{t+1}=\frac{1}{1+N_{t+1}}$. This defines the function $\alpha_{t}=\alpha\left(N_{t}\right)$ with $\frac{\partial \alpha\left(N_{t}\right)}{\partial N_{t}}<$ 0 . Note also that $\alpha_{t+1} \in(0,1] \Leftrightarrow N_{t+1} \in[0, \infty)$. Moreover, we can now define a sequence of random variables $\left\{N_{i}\right\}_{i=1}^{t}$, the initial value of which is exogenously given as $N_{1}=\frac{1-\alpha_{1}}{\alpha_{1}}$. Finally define $r\left(N_{t}\right) \equiv w\left(\alpha\left(N_{t}\right)\right)$, where $\frac{\partial r}{\partial N_{t}}=\frac{\partial w}{\partial \alpha_{t}} \frac{\partial \alpha_{t}}{\partial N_{t}}<0$ is readily established. It is also easy to see that $r(0)=w(1)>0$ and that $\lim _{N_{t} \rightarrow \infty} r\left(N_{t}\right)=w(0)<0$. Thus, for $\tilde{\tau} \in\left[\tau_{A}^{m}, \tau_{B}^{m}\right]$, there is a unique $\tilde{N}$ such that $r(\tilde{N})=0$. In light of these new definitions,

$$
N_{t+1}=N_{1} \cdot \frac{\phi\left(r\left(N_{1}\right)+\varepsilon_{1}\right)}{\phi\left(\varepsilon_{1}\right)} \cdot \frac{\phi\left(r\left(N_{2}\right)+\varepsilon_{2}\right)}{\phi\left(\varepsilon_{2}\right)} \cdot \ldots \cdot \frac{\phi\left(r\left(N_{t}\right)+\varepsilon_{t}\right)}{\phi\left(\varepsilon_{t}\right)}=N_{t} \cdot \frac{\phi\left(r\left(N_{t}\right)+\varepsilon_{t}\right)}{\phi\left(\varepsilon_{t}\right)}
$$

Notice that (8) is a non-linear stochastic first-order difference equation.

Observe first that if the sequence takes either the value 0 or the value $\tilde{N}$, it will take this value forever. This becomes immediate for $N_{t}=0$ by inserting $N_{t}=0$ into (8). For $N_{t}=\tilde{N}, r(\tilde{N})=0$ implies that $\frac{\phi\left(r\left(N_{t}\right)+\varepsilon_{t}\right)}{\phi\left(\varepsilon_{t}\right)}=\frac{\phi\left(\varepsilon_{t}\right)}{\phi\left(\varepsilon_{t}\right)}=1$, implying in turn $N_{t+1}=\tilde{N}$. If $N_{t}$ is infinity, $N_{t+1}$ will be too, since $\lim _{N_{t} \rightarrow \infty} r\left(N_{t}\right)$ is a finite negative number.

Note also that the sequence $\left\{N_{t}\right\}$ is a martingale. The reason is first that

$$
\begin{aligned}
E\left[N_{t+1}\right]= & \int_{-\infty}^{\infty} \ldots \int_{-\infty}^{\infty} N_{t+1} \cdot \phi\left(\varepsilon_{1}, \ldots, \varepsilon_{t}\right) d \varepsilon_{1} \ldots d \varepsilon_{t} \\
& =\int_{-\infty}^{\infty} \ldots \int_{-\infty}^{\infty} N_{1} \cdot \phi\left(r\left(N_{1}\right)+\varepsilon_{1}\right) \cdot \ldots \cdot \phi\left(r\left(N_{t}\right)+\varepsilon_{t}\right) d \varepsilon_{1} \ldots d \varepsilon_{t}=N_{1}<\infty,
\end{aligned}
$$


where the joint normal $\phi\left(\varepsilon_{1}, \varepsilon_{2}, \ldots, \varepsilon_{t}\right)=\phi\left(\varepsilon_{1}\right) \cdot \phi\left(\varepsilon_{2}\right) \cdot \ldots \cdot \phi\left(\varepsilon_{t}\right)$ by independence. Second, $E\left[N_{t+1} \mid\left\{N_{i}\right\}_{i=1}^{t}\right]=N_{t} \int_{-\infty}^{\infty} \frac{\phi\left(r\left(N_{t}\right)+\varepsilon_{t}\right)}{\phi\left(\varepsilon_{t}\right)} \phi\left(\varepsilon_{t}\right) d \varepsilon_{t}=N_{t} \int_{-\infty}^{\infty} \phi\left(r\left(N_{t}\right)+\varepsilon_{t}\right) d \varepsilon_{t}=N_{t}$. The martingale convergence theorem (e.g., Durrett, 2005, p. 233) states that $\left\{N_{t}\right\}$ converges almost surely to a limit $N_{\infty}$ with $E\left[N_{\infty}\right]<\infty$. For the interpretation of our model, it is necessary to evaluate the random variable $N_{\infty}$. Lemma 1 states that the martingale either converges towards 0 or towards $\tilde{N}$.

Lemma 1 The support of the random variable $N_{\infty}$ is $\{0, \tilde{N}\}$.

Proof. From the observation we made above, we know that $\operatorname{Pr}\left(N_{t+1}=0 \mid N_{t}=0\right)=1$ and $\operatorname{Pr}\left(N_{t+1}=\tilde{N} \mid N_{t}=\tilde{N}\right)=1$. We now prove by contradiction that there exists no other value $C$ the martingale $N_{t}$ can converge to. Note that the martingale convergence theorem directly states that $N_{t}$ cannot converge to infinity.

Assume there exists a number $C \in(0, \infty)$ where $N_{t}$ can converge to. Then, for every $\delta \in \mathbb{R}$ such that $0 \notin[C-\delta, C+\delta]$ and $\tilde{N} \notin[C-\delta, C+\delta]$, there exists a time period $t_{\delta}$, for which we have $N_{t_{\delta}+i} \in[C-\delta, C+\delta]$ for $i=0,1, \ldots$. Note that $\delta$ can be chosen arbitrarily small. Now define the variable $\bar{\varepsilon}_{t_{\delta}+i}$ by

$$
\bar{\varepsilon}_{t_{\delta}+i} \equiv \frac{\sigma^{2}}{r\left(N_{t_{\delta}+i}\right)} \cdot \ln \frac{N_{t_{\delta}+i}}{C+\delta}-\frac{1}{2} r\left(N_{t_{\delta}+i}\right)
$$

Note that $\bar{\varepsilon}_{t_{\delta}+i}$ is a shock such that $N_{t_{\delta}+i+1}=C+\delta$. Assume that $C<\tilde{N}$. Then, the variable $\bar{\varepsilon}_{t_{\delta}+i}$ is negative and finite for all $N_{t_{\delta}+i} \in[C-\delta, C+\delta]$, because all terms in (9) are finite. Therefore, for every $N_{t_{\delta}+i} \in[C-\delta, C+\delta], \operatorname{Pr}\left(\varepsilon_{t_{\delta}+i}<\bar{\varepsilon}_{t_{\delta}+i}\right)=\Phi\left(\bar{\varepsilon}_{t_{\delta}+i}\right)>0$, which means that the probability to draw an $\varepsilon_{t_{\delta}+i}<\bar{\varepsilon}_{t_{\delta}+i}$ is strictly positive for every $N_{t_{\delta}+i} \in[C-\delta, C+\delta]$. Thus, with a positive probability we observe an $N_{t_{\delta}+i+1}>$ 
$C+\delta$ for every period $t_{\delta}+i$ because $N_{t_{\delta}+i+1}$ depends negatively on $\varepsilon_{t_{\delta}+i}$. This means, that $\inf _{N_{t_{\delta}+i} \in[C-\delta, C+\delta]} \operatorname{Pr}\left(N_{t_{\delta}+i+1} \notin[C-\delta, C+\delta]\right)>0$, which is a contradiction to the assumption of convergence of $N_{t}$. Hence, $N_{t}$ cannot converge to $C$.

In order to prove non-convergence towards a $C>\tilde{N}$, we define $\underline{\varepsilon}_{t_{\delta}+i}$ as $\underline{\varepsilon}_{t_{\delta}+i} \equiv$ $\frac{\sigma^{2}}{r\left(N_{t_{\delta}+i}\right)} \cdot \ln \frac{N_{t_{\delta}+i}}{C-\delta}-\frac{1}{2} r\left(N_{t_{\delta}+i}\right)$ and use the equivalent reasoning as above.

We are now only left to show that the probability of $N_{t}$ converging to the set union of all $C$ is still 0. By choosing intervals around $C$ with rational endpoints, the probabilities can be summed up for the union set. Since we can choose $\delta$ arbitrarily, it is always possible to find an interval with rational endpoints for all $C$. Therefore, the sum of probabilities over these intervals is 0 . This completes the proof of Lemma 1.

From Slutzki's Theorem we know that if $N_{t}$ converges to $N_{\infty}$ with support $\{0, \tilde{N}\}$ almost surely, then $\alpha_{t}$ converges to $\alpha_{\infty}$ with support $\{\tilde{\alpha}, 1\}$ almost surely. For the belief $\alpha_{t}=1$ the tax rate $\tau_{A}^{m}$ is implemented, for $\tilde{\alpha}$ it is $\tilde{\tau}$. Therefore, the support of $\tau_{\infty}$ is $\left\{\tau_{A}^{m}, \tilde{\tau}\right\}$. This completes the proof of Proposition 2.

Proof of Corollary 2 It is clear that if $H_{A}(\tau) \neq H_{B}(\tau)$ for all $\tau \in\left[\tau_{A}^{m}, \tau_{B}^{m}\right]$, then the function $s(\tau) \equiv H_{A}(\tau)-H_{B}(\tau)$ is never equal to zero in the relevant interval. Consequently, the functions $\omega\left(\alpha_{t}\right)$ and $r\left(N_{t}\right)$ defined in the proof of Proposition 2 will also be non-zero in the relevant range. Therefore, equation (8) has a unique fixed point, which is $N_{t+1}=N_{t}=0$, corresponding to $\alpha_{t+1}=\alpha_{t}=1$.

Proof of Proposition 3 From Proposition 2 we know that $\alpha_{t}$ either converges to 1 or to $\tilde{\alpha}$. What we need to characterize in order to prove Proposition 3 is actually the 
distribution of the random variable $N_{\infty}$ over $\{0, \tilde{N}\}$, from which we can then deduce the distribution of the random variable $\alpha_{\infty}$ over $\{1, \tilde{\alpha}\}$. Corollary 2.11 in Durrett $(2005)$ implies that $E\left[N_{\infty}\right] \leq E\left[N_{1}\right]$. Let $\mu$ be the probability of convergence towards $\tilde{N}$. Then $E\left[N_{\infty}\right]=(1-\mu) \cdot 0+\mu \cdot \tilde{N}=\mu \cdot \tilde{N}$, which implies $\mu \leq \frac{N_{1}}{\tilde{N}}$ and hence $(1-\mu) \geq 1-\frac{N_{1}}{\tilde{N}}$, where it will be recalled that $N_{1}=\frac{1-\alpha_{1}}{\alpha_{1}}$ and hence $E\left[N_{1}\right]=N_{1}$. As it is a probability, $\xi$ must be nonnegative. It equals the minimum value of $(1-\mu)$ if $(1-\mu)>0$. Hence,

$$
\xi=\max \left\{0,1-\frac{N_{1}}{\tilde{N}}\right\}=\max \left\{0,1-\frac{\frac{1-\alpha_{1}}{\alpha_{1}}}{\frac{1-\tilde{\alpha}}{\tilde{\alpha}}}\right\}=\max \left\{0, \frac{\alpha_{1}-\tilde{\alpha}}{\alpha_{1}(1-\tilde{\alpha})}\right\}
$$

\section{References}

Acemoglu, D., And J. A. Robinson (2000): "Why Did the West Extend the Franchise? Democracy, Inequality, and Growth in Historical Perspective," Quarterly Journal of Economics, 115, 1167-1199.

Alesina, A., And G.-M. Angeletos (2005): "Fairness and Redistribution," American Economic Review, 95(4), 960-80.

BanerJee, A. V. (1992): “A Simple Model of Herd Behavior," Quarterly Journal of Economics, 107(3), 797-817.

Baron, D. P. (1996): "A Dynamic Theory of Collective Goods Programs," The American Political Science Review, 90(2), 316-330.

Bartels, L. M. (1996): "Uninformed Votes: Information Effects in Presidential Eleections," American Journal of Political Science, 40(1), 194-230. 
BAuer, P. T. (1981): Equality, the Third World, and Economic Delusion. Harvard University Press.

Bikhchandani, S., D. Hirshleifer, and I. Welch (1992): "A Theory of Fads, Fashion, Custom, and Cultural Change in Informational Cascades," Journal of Political Economy, 100(5), 992-1026.

Blendon, R. J., J. M. Benson, M. Brodie, R. Morin, D. E. Altman, D. Gitterman, M. Brossard, and M. James (1997): "Bridging the Gap between the Public's and Economists Views of the Economy," Journal of Economic Perspectives, 11(3), 105-118.

Crawford, V. P., And J. Sobel (1982): "Strategic Information Transmission," Econometrica, 50(6), 1431-1451.

Durrett, R. (2005): Probability: Theory and Examples. Curt Hinrichs.

Easley, D., And N. M. Kiefer (1988): "Controlling a Stochastic Process with Unknown Parameters," Econometrica, 56(5), 1045-64.

Easterly, William, S. (2001): The Elusive Quest for Growth. MIT Press.

Feddersen, T. J., And W. Pesendorfer (1996): "The Swing Voter's Curse," American Economic Review, 86(3), 408-424.

Friedman, M. (1962): Capitalism and Freedom. University of Chicago Press.

(1997): "If Only the U.S. Were as Free as Hong Kong," Wall Street Journal, July 8, A14. 
Fuchs, V. R., A. S. Blinder, and J. M. Poterba (1998): "Economists' Views about Parameters, Values and Policies: Survey Results in Labor and Public Economics," Journal of Economic Literature, 36(3), 1387-1425.

Gans, J. S., And M. Smart (1996): "Majority Voting with Single-Crossing Preferences," Journal of Public Economics, 59(2), 219-237.

Hayek, F. A. (1944): The Road To Serfdom. Routledge.

Hazlitt, H. (1946): Economics in One Lesson. Harper \& Brothers.

Heidhues, P., And J. LAGerlöF (2003): "Hiding information in electoral competition," Games and Economic Behavior, 42, 48-74.

Hess, G. D., And A. Orphanides (2001): "War and Democracy," Journal of Political Economy, 109(4), 776-810.

Hogg, R., And A. T. CRAig (1995): Introduction to Mathematical Statistics. Englewood Cliffs, N.J. : Prentice Hall.

KAnt, I. (1784): "An Answer to the Question: What is Enlightenment?," http://www.english.upenn.edu/mgamer/Etexts/kant.html.

Laslier, J.-F., A. Trannoy, and K. Van Der Straeten (2003): "Voting under ignorance of job skills of unemployed: the overtaxation bias," Journal of Public Economics, 87(3-4), 595-626.

McLennan, A. (1984): "Price Dispersion and Incomplete Learning in the Long Run," Journal of Economic Dynamics and Control, 7(3), 331-47. 
Myrdal, G. (1975): "The Equality Issue in World Development: Nobel Memorial Lecture," Scnadinavian Journal of Economics, pp. 413-432.

Persson, T., and G. Tabellini (2000): Political Economics. Mit Press, Masschusetts.

Piketty, T. (1995): "Social Mobility and Redistributive Politics," Quarterly Journal of Economics, 110(3), 551-84.

Putnam, R. D. (1993): Making Democracy Work. Princeton University Press.

Rosenstein-Rodan, P. (1943): "Problems of Industrialization of Eastern and SouthEastern Europe," Economic Journal, pp. 202-211.

Sachs, J. (2005): The End of Poverty: Economic Possibilities for Our Time. The Penguin Press.

Schultz, C. (1996): "Polarization and Inefficient Policies," Review of Economci Studies, 63(2), 331-44.

Spector, D. (2000): "Rational Debate and One-Dimensional Conflict," Quarterly Journal of Economics, 115(1), 181-200.

Wittman, D. (1989): "Why Democracies produce efficient results?," Journal of Political Economy, pp. 1395-1424. 